\title{
Continuous Administration of Vancomycin through a Long Intestinal Tube for Clostridium difficile Infection
}

\author{
Naoto Mizumura, Koichi Demura, Masayasu Kawasaki, Satoshi Okumura, Sho Toyoda, \\ Atsuo Imagawa, Masao Ogawa, Kazuki Ohba and Masao Kameyama
}

\begin{abstract}
A 76-year-old previously healthy Japanese man experienced severe diarrhea $(8,000 \mathrm{~mL}$ per day) after undergoing laparotomy for small bowel obstruction and was diagnosed with Clostridium difficile infection. Although he developed a maculopapular rash secondary to the systemic absorption of enteral vancomycin (VCM), the patient was successfully treated with the continuous administration of VCM through a long intestinal tube placed in the terminal ileum. This method ensured the reliable delivery of VCM to the colon, while the continuous administration maintained high fecal levels of the drug. This treatment approach is an effective minimally invasive option for patients with severe diarrhea.
\end{abstract}

Key words: continuous administration, Clostridium difficile, vancomycin, long intestinal tube, maculopapular rash, systemic absorption

(Intern Med 54: 1559-1562, 2015)

(DOI: 10.2169/internalmedicine.54.4026)

\section{Introduction}

Clostridium difficile infection (CDI) is the most frequent cause of hospital-acquired diarrhea. The clinical manifestations of CDI range from asymptomatic carriage, to mild diarrhea and fulminant colitis (1). Patients with severe complicated CDI are treated with oral vancomycin (VCM) and intravenous metronidazole (MNZ). If their condition does not improve with medical therapy, subtotal colectomy may be considered (1-3). In Japan, intravenous MNZ was not licensed for use until May 2014, and subtotal colectomy for CDI is not common. In contrast, oral VCM is the most widely used treatment for CDI in Japan. To our knowledge, there have been no previous reports of treatment with the continuous infusion of enteral VCM. We herein report a case of CDI with large-volume diarrhea successfully treated with the continuous administration of VCM via a transnasal ileus tube.

\section{Case Report}

In December 2013, a 76-year-old Japanese man with no significant past medical history was admitted to our hospital with a diagnosis of small bowel obstruction. On a physical examination, his temperature was $37.3^{\circ} \mathrm{C}$, his heart rate was 98 beats/min, his blood pressure was $138 / 78 \mathrm{mmHg}$ and his oxygen saturation was $98 \%$ on ambient air. He presented with diffuse abdominal distension and tenderness, although no signs of peritoneal irritation were noted. Laboratory tests revealed a white blood cell count of 5,400 cells $/ \mathrm{mm}^{3}$, Creactive protein level of $0.43 \mathrm{mg} / \mathrm{dL}$ and normal hepatic and renal functions. A contrast-enhanced computed tomography (CT) scan of the abdomen and pelvis showed dilatation of the small bowel with a caliber change in the terminal ileum. A long transnasal intestinal tube was inserted for bowel decompression, and the administration of cefmetazole was started at a dose of $1 \mathrm{~g}$ twice a day. However, decompression was not achieved successfully, and laparotomy was subsequently performed on hospital day 3. After exploring the peritoneal cavity, stenosis and adhesion were identified in the terminal ileum, and adhesiolysis was performed without bowel resection. The tip of the long intestinal tube was placed in the terminal ileum during the operation (Fig. 1). Postoperatively, the patient exhibited a persistently high fever, and cefmetazole was changed to ceftriaxone at a dose

Department of Surgery, Bellland General Hospital, Japan

Received for publication September 2, 2014; Accepted for publication October 29, 2014

Correspondence to Dr. Naoto Mizumura, n_mizumura@seichokai.or.jp 


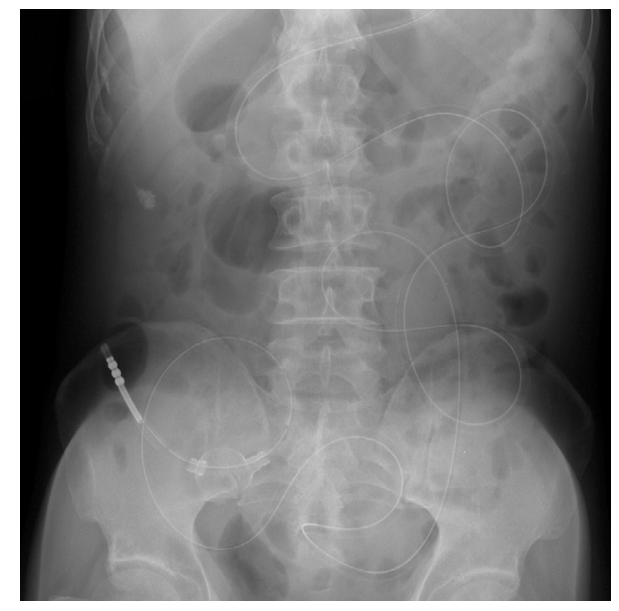

Figure 1. Abdominal radiograph showing the tip of the long intestinal tube placed in the terminal ileum.

of $1 \mathrm{~g}$ twice a day on hospital day 6 .

On day 9 , the patient developed watery diarrhea 12 times a day and was diagnosed with CDI based on an enzyme immunoassay for $C$. difficile glutamate dehydrogenase and toxins A and B (C. DIFF QUIK CHEK COMPLETE; Alere). The administration of ceftriaxone was immediately stopped, and treatment for CDI with VCM $(500 \mathrm{mg})$ was initiated four times a day through the long intestinal tube. On day 10 , he was admitted to the intensive care unit due to hypovolemic shock (Acute Physiology and Chronic Health Evaluation II score: 14; Sequential Organ Failure Assessment score: 2). His temperature was $38.0^{\circ} \mathrm{C}$, his heart rate was 114 beats/min and his blood pressure was $82 / 59$ $\mathrm{mmHg}$. Laboratory tests showed a white blood cell count of 8,600 cells $/ \mathrm{mm}^{3}$, C-reactive protein level of $13.75 \mathrm{mg} / \mathrm{dL}$, serum albumin level of $2.7 \mathrm{~g} / \mathrm{dL}$, serum creatinine level of $1.72 \mathrm{mg} / \mathrm{dL}$ and serum lactate level of $2 \mathrm{mmol} / \mathrm{L}$. A noncontrast CT scan showed the large bowel to be filled with fluid in addition to thickening of the wall of the sigmoid colon (Fig. 2). A rectal catheter (Flexi-Seal; ConvaTec Japan, Tokyo, Japan) was inserted to measure the volume of diarrhea. The patient subsequently passed $8,000 \mathrm{~mL}$ of diarrhea and was given $10,000 \mathrm{~mL}$ of lactated Ringer's solution the same day. Although he became hemodynamically stable and the drainage volume through the long intestinal tube was $\leq 200 \mathrm{~mL} /$ day, the reduction in stool output achieved after five days of VCM therapy was insufficient (Fig. 3). On day 14 , the treatment was changed to the continuous administration of VCM (saline $100 \mathrm{~mL}+\mathrm{VCM} 500 \mathrm{mg}$; for six hours four times a day) through the intestinal tube, and the stool output decreased over the next few days.

On day 18 , the patient's temperature rose to $39.1^{\circ} \mathrm{C}$. A contrast-enhanced CT scan showed multiple wedge-shaped and low-density lesions in the right kidney. He was therefore diagnosed with right pyelonephritis, and Pseudomonas aeruginosa was subsequently identified in urine cultures. Cefepime $(1 \mathrm{~g})$ and tobramycin $(240 \mathrm{mg})$ were administered, after which a faint maculopapular rash developed on

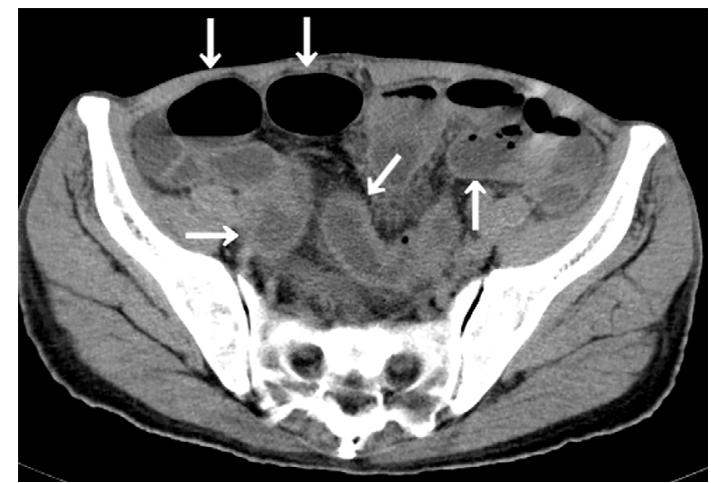

Figure 2. Non-contrast CT scan of the abdomen and pelvis. White arrows show the thickening of the wall of the sigmoid colon.

the limbs. The antibiotic therapy was immediately changed to tazobactam/piperacillin ( $4.5 \mathrm{~g}$ three times a day); however, the rash gradually spread to the face and trunk (Fig. 4). On day 22, after 13 days of VCM therapy, the serum VCM concentration was $3.9 \mu \mathrm{g} / \mathrm{mL}$; the administration of VCM was thus stopped immediately. The patient was then administered a topical corticosteroid and oral antihistamine, and the rash resolved over the next few days. He thereafter exhibited a good recovery and was discharged on day 31 .

\section{Discussion}

The diagnosis of CDI is made based on a combination of particular signs and symptoms confirmed according to microbiological evidence of $C$. difficile toxin and toxinproducing $C$. difficile in the stool (1-3). In the current case, although methicillin-resistant Staphylococcus aureus (MRSA) was isolated from a stool culture, the patient was diagnosed with CDI based on the results of an enzyme immunoassay for $C$. difficile glutamate dehydrogenase and toxins $A$ and $B(4)$.

Classifying the severity of colitis is important for determining the appropriate treatment regimen for CDI. The present patient met the criteria for severe complicated CDI $(1,2)$. The treatment of severe complicated CDI involves VCM delivered orally or via an enteric feeding tube (125 mg or $500 \mathrm{mg}$ four times per day) and MNZ administered intravenously (500 mg three times a day) $(1,2)$. Because diarrhea results in significant volume depletion, providing supportive care with intravenous fluid resuscitation and electrolyte replacement is also important. In the current case, a rectal catheter was useful for determining the quantity of fluid loss.

Treatment for CDI with VCM was initiated at a dose of $500 \mathrm{mg}$ four times a day through the long intestinal tube; however, the patient failed to improve after five days of VCM therapy (Fig. 3). The time to resolution of diarrhea in patients treated with VCM using a regimen of $500 \mathrm{mg}$ four times a day is 2.8 days (5), and most VCM-treated patients 


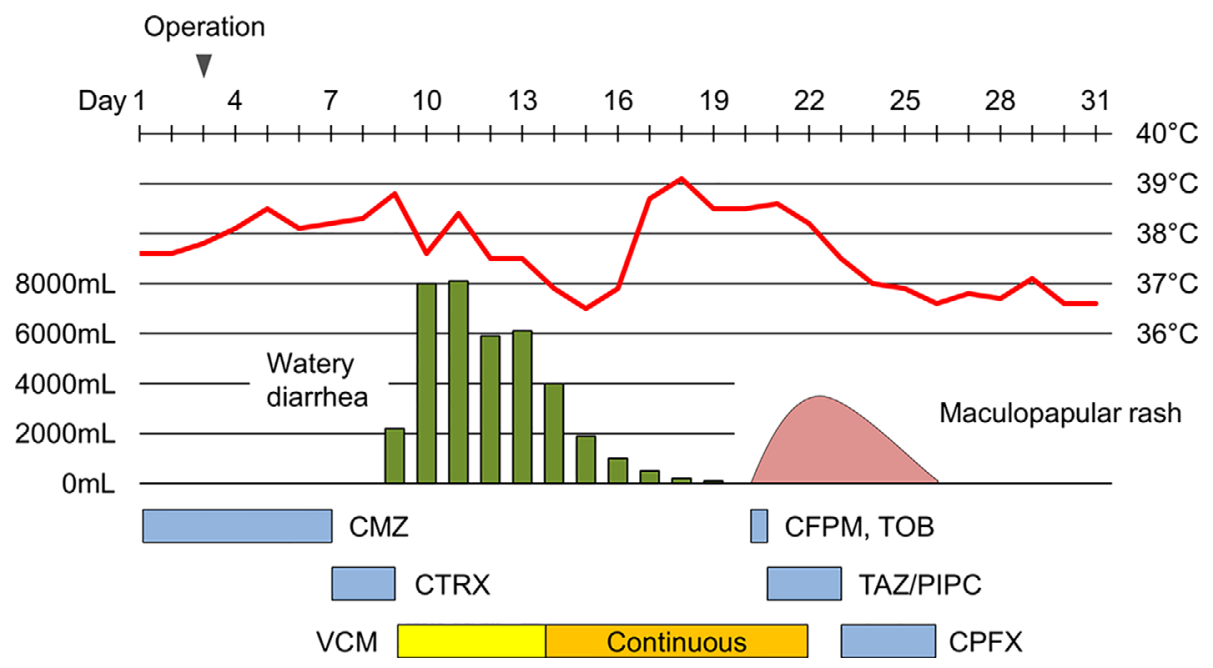

Figure 3. Clinical course of the patient. CMZ: cefmetazole (1 $\mathrm{g}$ twice a day), CTRX: ceftriaxone (1 g twice a day), VCM: vancomycin ( $2 \mathrm{~g}$ a day), CFPM: cefepime (a single dose of $1 \mathrm{~g}$ ), TOB: tobramycin (a single dose of $240 \mathrm{mg}$ ), TAZ/PIPC: tazobactam/piperacillin (4.5 g thrice a day), CPFX: ciprofloxacin (300 $\mathrm{mg}$ twice a day)

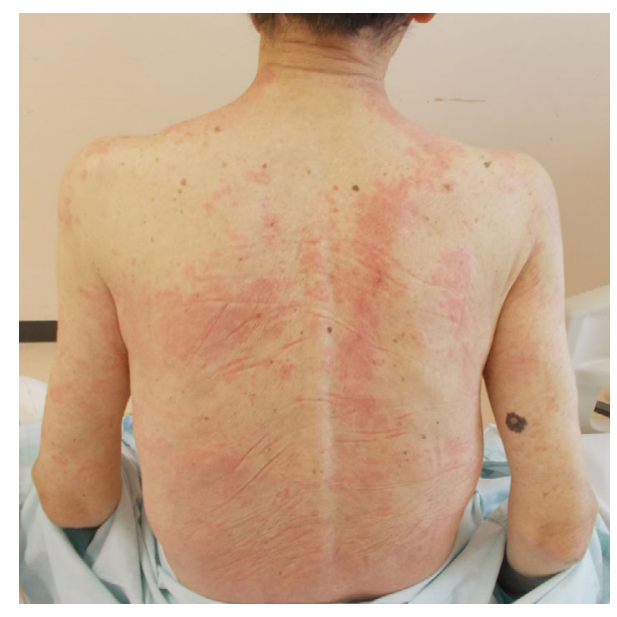

Figure 4. Image of the maculopapular rash on the patient's back.

exhibit resolution of diarrhea within five days $(6,7)$. Therefore, if the patient fails to improve within five days, surgical intervention may be considered (1). Recently, diverting loop ileostomy and colonic lavage have been shown to reduce mortality compared with that observed in historical controls treated with colectomy (19\% versus 50\%). This surgical approach involves loop ileostomy, intraoperative colonic lavage and postoperative antegrade instillation of VCM flushes via the ileostomy (8). The success of this technique highlights the importance of reliably administering VCM to the colon. In our patient, VCM was reliably delivered into the colon via the tube placed in the terminal ileum, as can be achieved via ileostomy; however, this treatment resulted in only a slight decrease in stool output. Therefore, subtotal colectomy was considered. Nevertheless, the 30-day postoperative mortality rate is high $(41.3 \%)$ in patients with severe CDI treated with emergency surgery (9). Furthermore, subtotal colectomy is rarely performed for CDI in Japan, and our search of the Japan Medical Abstracts Society (1974-2013) identified only four cases.

We suspected that the VCM was being expelled just after administration in the $300 \mathrm{~mL}$ of diarrhea passed by the patient each hour. In other words, the residence time of VCM on the surface of the colon was extremely short. With the patient's consent, the intermittent VCM therapy was changed to continuous administration via the intestinal tube. Consequently, he displayed no symptoms of abdominal distension or vomiting during the continuous treatment, and his stool output decreased over the next few days (Fig. 3).

The fecal level of VCM is affected by the stool frequency (10). In the present case, the fecal VCM concentration following continuous administration reached very high levels (due to the presence of the watery component, the fecal samples were centrifuged and the concentration of the supernatant was measured by SRL Inc. using an enzyme immunoassay), as follows: $554 \mu \mathrm{g} / \mathrm{mL}$ on day $14(4,000 \mathrm{~mL}$ of diarrhea per day) and $1,127 \mu \mathrm{g} / \mathrm{mL}$ on day $15(2,000 \mathrm{~mL}$ of diarrhea per day). Indeed, the total daily level of fecal excretion of VCM was almost equal to the daily dose of 2,000 $\mathrm{mg}$. The minimum inhibitory concentration (MIC) of VCM for $C$. difficile is $0.5-4.0 \mu \mathrm{g} / \mathrm{mL}$ (11). Although the effective fecal concentration of VCM is unclear, the fecal VCM level in this case was maintained at several hundred times the MIC for C. difficile.

Unfortunately, a maculopapular rash appeared after 11 days of VCM therapy. Oral VCM is poorly absorbed; however, bowel inflammation may enhance the absorption of oral VCM (12). The accumulation of oral VCM is most often reported in patients with renal insufficiency, although it can also occur in patients with a normal renal function (12-14). On day 22, our patient had a normal renal function (serum creatinine: $0.68 \mathrm{mg} / \mathrm{dL}$ ). Nevertheless, the 
high dose of VCM induced a high serum VCM concentration $(3.9 \mu \mathrm{g} / \mathrm{mL})$. The systemic absorption of oral VCM is known to cause adverse effects, including maculopapular rashes $(15,16)$, red man syndrome $(13,17,18)$, anaphylaxis (19), leukopenia (14) and encephalopathy (20). Based on these findings and the patient's clinical course, we suspected that he developed the maculopapular rash secondary to VCM (21). Tests for identifying the causative drug were subsequently conducted; the drug-induced lymphocyte stimulation test was positive for VCM but negative for cefepime and tobramycin, and patch testing was negative for VCM. Although the continuous administration of VCM may have contributed to increased drug absorption in this case, it is important to monitor the serum VCM concentration regardless of the renal function, particularly in patients exhibiting such adverse effects.

The current patient was successfully treated with the continuous administration of VCM through a long intestinal tube, thus avoiding the need for subtotal colectomy. The VCM was reliably delivered into the colon via the tube placed in the terminal ileum, and the continuous administration maintained high fecal levels of the drug. Although further research is needed to confirm the safety and efficacy of this treatment, this technique may be an effective minimally invasive treatment option for patients with severe diarrhea.

The authors state that they have no Conflict of Interest (COI).

Written informed consent was obtained from the patient for the publication of this case report.

\section{Acknowledgement}

We thank Crimson Interactive Pvt. Ltd for their assistance in translating the manuscript.

\section{References}

1. Surawicz CM, Brandt LJ, Binion DG, et al. Guidelines for diagnosis, treatment, and prevention of Clostridium difficile infections. Am J Gastroenterol 108: 478-498, 2013.

2. Cohen SH, Gerding DN, Johnson S, et al. Clinical practice guidelines for Clostridium difficile infection in adults: 2010 update by the society for healthcare epidemiology of America (SHEA) and the infectious diseases society of America (IDSA). Infect Control Hosp Epidemiol 31: 431-455, 2010.

3. Debast SB, Bauer MP, Kuijper EJ. European Society of Clinical Microbiology and Infectious Diseases: update of the treatment guidance document for Clostridium difficile infection. Clin Microbiol Infect 20(Suppl 2): 1-26, 2014.

4. Iwata K, Doi A, Fukuchi T, et al. A systematic review for pursuing the presence of antibiotic associated enterocolitis caused by methicillin resistant Staphylococcus aureus. BMC Infect Dis 14:
247, 2014

5. Teasley DG, Gerding DN, Olson MM, et al. Prospective randomised trial of metronidazole versus vancomycin for Clostridiumdifficile-associated diarrhoea and colitis. Lancet 2: 1043-1046, 1983.

6. Zar FA, Bakkanagari SR, Moorthi KM, Davis MB. A comparison of vancomycin and metronidazole for the treatment of Clostridium difficile-associated diarrhea, stratified by disease severity. Clin Infect Dis 45: 302-307, 2007.

7. Al-nassir WN, Sethi AK, Nerandzic MM, Bobulsky GS, Jump RL, Donskey CJ. Comparison of clinical and microbiological response to treatment of Clostridium difficile-associated disease with metronidazole and vancomycin. Clin Infect Dis 47: 56-62, 2008.

8. Neal MD, Alverdy JC, Hall DE, Simmons RL, Zuckerbraun BS. Diverting loop ileostomy and colonic lavage: an alternative to total abdominal colectomy for the treatment of severe, complicated Clostridium difficile associated disease. Ann Surg 254: 423-427, 2011.

9. Bhangu A, Nepogodiev D, Gupta A, Torrance A, Singh P. Systematic review and meta-analysis of outcomes following emergency surgery for Clostridium difficile colitis. Br J Surg 99: 1501-1513, 2012.

10. Gonzales M, Pepin J, Frost EH, et al. Faecal pharmacokinetics of orally administered vancomycin in patients with suspected Clostridium difficile infection. BMC Infect Dis 10: 363, 2010.

11. Drummond LJ, Mccoubrey J, Smith DG, Starr JM, Poxton IR. Changes in sensitivity patterns to selected antibiotics in Clostridium difficile in geriatric in-patients over an 18-month period. $\mathbf{J}$ Med Microbiol 52: 259-263, 2003.

12. Armstrong CJ, Wilson TS. Systemic absorption of vancomycin. J Clin Pathol 48: 689, 1995.

13. Bergeron L, Boucher FD. Possible red-man syndrome associated with systemic absorption of oral vancomycin in a child with normal renal function. Ann Pharmacother 28: 581-584, 1994.

14. Aradhyula S, Manian FA, Hafidh SA, Bhutto SS, Alpert MA. Significant absorption of oral vancomycin in a patient with Clostridium difficile colitis and normal renal function. South Med J 99: 518-520, 2006.

15. McCullough JM, Dielman DG, Peery D. Oral vancomycin-induced rash: case report and review of the literature. DICP 25: 13261328, 1991.

16. Osawa R, Kaka AS. Maculopapular rash induced by oral vancomycin. Clin Infect Dis 47: 860-861, 2008.

17. Rao S, Kupfer Y, Pagala M, Chapnick E, Tessler S. Systemic absorption of oral vancomycin in patients with Clostridium difficile infection. Scand J Infect Dis 43: 386-388, 2011.

18. Nallasivan M, Maher F, Murthy K. Rare case of "red man" syndrome in a female patient treated with oral vancomycin for Clostridium difficile diarrhoea. BMJ Case Rep, 2009.

19. Bossé D, Lemire C, Ruel J, Cantin AM, Ménard F, Valiquette L. Severe anaphylaxis caused by orally administered vancomycin to a patient with Clostridium difficile infection. Infection 41: 579-582, 2013.

20. Thompson CM, Long SS, Gilligan PH, Prebis JW. Absorption of oral vancomycin: possible associated toxicity. Int J Pediatr Nephrol 4: 1-4, 1983.

21. Stern RS. Clinical practice. Exanthematous drug eruptions. N Engl J Med 366: 2492-2501, 2012.

(C) 2015 The Japanese Society of Internal Medicine http://www.naika.or.jp/imonline/index.html 